\title{
Contrast-free percutaneous pulmonary valve replacement: a safe approach for valve-in-valve procedures
}

\author{
Barry O'Callaghan, Jenny Zablah, Ryan Leahy, Michael Shorofsky, Joseph Kay, Gareth Morgan
}

The Heart Institute, Children's Hospital Colorado, Aurora, USA

Adv Interv Cardiol 2021; 17, 2 (64): 200-209

DOI: https://doi.org/10.5114/aic.2021.107500

\begin{abstract}
A bstract
Introduction: Percutaneous pulmonary valve replacement (PPVI) continues to gather pace in pediatric and adult congenital practice. This is fueled by an expanding repertoire of devices, techniques and equipment to suit the heterogenous anatomical landscape of patients with lesions of the right ventricular outflow tract (RVOT). Contrast-induced nephropathy is a real risk for teenagers and adults with congenital heart disease (CHD).

Aim: To present a series of patients who underwent PPVI without formal RVOT angiography and propose case selection criteria for patients who may safely benefit from this approach.

Material and methods: We retrospectively collected PPVI data from the preceding 2 years at our institution identifying patients who had been listed as suitable for consideration for contrast-free PPVI from our multidisciplinary team (MDT) meeting based on predefined criteria. Demographic, clinical, imaging and hemodynamic data were collected. Data were analyzed using SPSS.

Results: Twenty-one patients were identified. All patients had a technically successful implantation with improvements seen in invasive and echocardiographic hemodynamic measurements. $90 \%$ of patients had a bio-prosthetic valve (BPV) in situ prior to PPVI. One patient had a complication which may have been recognized earlier with post-intervention RVOT contrast injection.

Conclusions: Zero-contrast PPVI is technically feasible and the suitability criteria for those who might benefit are potentially straightforward. The advent of fusion and 3D imaging in cardiac catheterization laboratories is likely to expand our capacity to perform more procedures with less contrast. Patients with bio-prosthetic valves in the pulmonary position may benefit from contrast-free percutaneous pulmonary valve implantation.
\end{abstract}

Key words: congenital heart disease, tetralogy of Fallot, percutaneous pulmonary valve implantation, pediatric cardiac catheterization, adult congenital heart disease.

Su m m a ry

This paper provides an overview of our institutional experience with percutaneous pulmonary valve replacement performed without the use of contrast to evaluate the right ventricular outflow tract. It presents a practical approach to the procedure and proposes potentially suitable patient candidates for such a procedure. It presents the relevant literature around fusion imaging and discusses the very relevant topic of reducing contrast use where possible in the cardiac catheterization laboratory, particularly as we acknowledge the increased age of the adult congenital heart disease patient population. It concludes that this approach may be considered for patients with bio-prosthetic valves in the pulmonary position, particularly when Sapien 3 valve implantation is being considered.

\section{Introduction}

Right ventricular outflow tract (RVOT) dysfunction is a common endpoint for congenital heart patients, particularly those with tetralogy of Fallot and its variants who have undergone prior intervention in early childhood [1]. A variety of interventions exist to palliate the circulation long term including surgically implanted bioprosthetic valves of various constructs. Almost all such implants fail due to degeneration of foreign tissue. Percutaneous pulmonary valve implantation has become the treatment of choice for the dysfunctional RVOT and the battery of inventory employable to treat patients continues to ex-

\section{Corresponding author:}

Gareth Morgan MB, BCh, BAO, MPhil, FSCAI, The Heart Institute, Children's Hospital Colorado, 13123 E 16th Ave, Aurora, CO 80045, USA, e-mail: gareth.morgan@childrenscolorado.org

Received: 6.10.2020, accepted: 4.03.2021. 
pand, increasing our capacity to provide this treatment for more patients with heterogeneous RVOT substrate.

Older children and adults with congenital heart disease suffer the compound ill effects of various interventions and altered physiology over a lifetime. These may include multiple renal insults. Ischemia reperfusion injury, cyanosis, polycythemia, hypoperfusion, nephrotoxic medications and radiographic contrast agents are some of the insults suffered by their developing nephrons. This is in addition to age-related changes in adult patients [2]. For this reason a low- or no-contrast approach to congenital catheterization, particularly in adults, should be considered in all possible instances, especially considering the expansion of fusion imaging and adjunct intraoperative imaging techniques that are available to the interventional cardiologist, some of which do not require the use of intravascular contrast.

We present a series of patients who have undergone a zero-contrast assessment of the RVOT at the time of percutaneous pulmonary valve implantation.

\section{Aim}

We aim to present a series of patients who underwent percutaneous pulmonary valve replacement (PPVI) without formal RVOT angiography at our institution over the last 10 years and propose case selection criteria for patients who may safely benefit from this approach to intervention. We aim to discuss the relevance of contrast-free percutaneous interventions on congenital heart patients due to their inherent risk for developing chronic kidney disease.

\section{Material and methods}

We reviewed all patients in our center who had undergone PPVI over the preceding 2 years, accounting for a change in practice whereby contrast-free PPVI had been offered in that time period. Patients had been identified at our multi-disciplinary team (MDT) meeting for consideration for contrast-free implantation of a percutaneous pulmonary valve based on predefined criteria. Criteria included presence of a bio-prosthetic valve (BPV), presence of a previously stented homograft or conduit and patients with dysfunctional RVOT and significant kidney dysfunction who had extensive pre-procedural cross sectional imaging for the purposes of procedural planning. Patients who did not have adequate cross sectional imaging for coronary compression risk evaluation had a low-contrast (<20 ml) evaluation of their coronary tree with balloon interrogation. We sought institutional review board (IRB) approval prior to undertaking this study. We identified patients to include for this evaluation by searching our institutional PPVI database and reviewing case notes of patients who had received less than $20 \mathrm{ml}$ of contrast during a PPVI. Those who had RVOT contrast evaluation on the day, either planned or unplanned, were excluded (Figure 1).

\section{Statistical analysis}

A variety of parameters were documented for analysis including demographic and clinical details inclusive of the patients' diagnoses and past surgical history. Hemodynamic and fluoroscopic data were collected from PedCath (Scientific Software Solutions). Echocardiographic parameters inclusive of transthoracic and intracardiac echocardiography were collected from Syngo (Siemens Medical Solutions, USA). Cross sectional imaging data and reports were reviewed on various imaging data operating platforms. Data were collected, cleaned and uploaded into SPSS v22 (IBM Corp.). Data are presented as means, medians, standard deviations and ranges where appropriate and all analysis was performed in SPSS v22 (IBM Corp). A $p$-value of $<0.05$ was considered statistically significant.

\section{Results}

\section{Baseline characteristics}

Nineteen patients had a previously surgically implanted bioprosthetic valve. Two had pulmonary homografts in the RVOT position. One of these had a dysfunctional $22 \mathrm{~mm}$ Melody Valve, previously implanted within the stented homograft. Baseline cohort characteristics are included in Table I. The 2 most commonly encountered

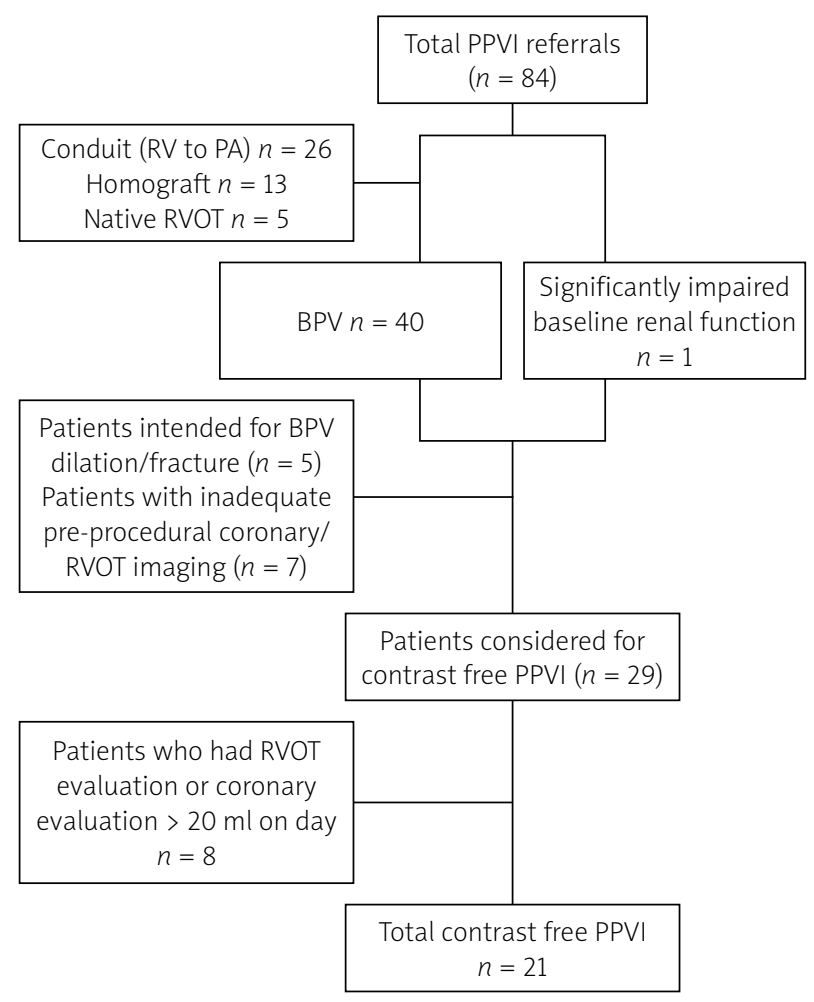

Figure 1. Patient identification, selection and exclusion for contrast-free evaluation

$P P V I$ - percutaneous pulmonary valve implantation, RVPA - right ventricular to pulmonary artery, BPV - bioprosthetic valve, RVOT - right ventricular outflow tract. 
valves were the Sorin Mitroflow $(n=13)$ and the Carpentier Edwards Perimount $(n=5)$. Median age was 27 years (8-58). The most common intracardiac lesion was tetralogy of Fallot and its variants $(n=15)$. The mean time from prosthetic valve implantation to PPVI was 8.5 years (6-17). The nature of valve dysfunction was isolated stenosis in 4 patients, with mixed disease (stenosis and regurgitation) encountered in the remaining patients. Thirty-eight percent of patients had severe PI prior to intervention. All but 1 patient had implantation of a Sapien 3 valve with $26 \mathrm{~mm}$ valves implanted in the majority $(n=12)$. One patient had a Melody PPVI (18 mm ensemble) placed within a Sorin Mitroflow valve $(21 \mathrm{~mm})$. Mean hospital stay was 29 h. All patients $(n=10)$ who underwent PPVI in our adult congenital catheterization laboratory had baseline pre- and post-procedural renal function assessed on blood biochemistry. One patient had abnormal pre-PPVI renal biochemistry with a history of acute kidney injury (pre-PPVI blood urea nitrogen (BUN) $47 \mathrm{mg} / \mathrm{dl}$, serum creatinine 1.51). All other patients had normal pre- and post-procedural renal biochemistry. Median BUN was $17.5 \mathrm{mg} / \mathrm{dl}$ (6-23) before the interven-

Table I. Cohort characteristics

\begin{tabular}{|c|c|}
\hline Parameter & Value \\
\hline Gender & M: 10, F: 11 \\
\hline \multirow[t]{4}{*}{ Primary diagnosis } & TOF + variants $(n=15)$ \\
\hline & Pulmonary stenosis $(n=3)$ \\
\hline & Pulmonary atresia $(n=2)$ \\
\hline & Ross procedure $(n=1)$ \\
\hline \multirow{8}{*}{$\begin{array}{l}\text { Pre-procedural } \\
\text { RVOT morphology/ } \\
\text { BPV type }\end{array}$} & Sorin Mitroflow $(n=13)$ \\
\hline & $21 \mathrm{~mm}(\mathrm{TID}, 17 \mathrm{~mm}), n=1$ \\
\hline & $25 \mathrm{~mm}$ (TID, $21 \mathrm{~mm}), n=1$ \\
\hline & $27 \mathrm{~mm}$ (TID, $23 \mathrm{~mm}), n=11$ \\
\hline & $\begin{array}{l}\text { Carpentier Edwards perimount (CE) }(n=5) \\
29 \mathrm{~mm}(\mathrm{TID}, 27 \mathrm{~mm}), n=5\end{array}$ \\
\hline & $\begin{array}{c}\text { Mosaic }(n=1) \\
27 \mathrm{~mm}(\text { TID, } 24 \mathrm{~mm}), n=1\end{array}$ \\
\hline & $\begin{array}{l}\text { Homograft ( } n=2, n=1 \text { with previous } \\
22 \mathrm{~mm} \text { melody PPVI) }\end{array}$ \\
\hline & $\begin{array}{c}\text { Median TID in } 19 \text { patients } \\
(23 \mathrm{~mm}(17-27 \mathrm{~mm})) \text {, see Figure } 1\end{array}$ \\
\hline $\begin{array}{l}\text { Time interval (BPVI } \\
\text { to PPVI) [years] }\end{array}$ & $8.5(6-17)$ \\
\hline $\begin{array}{l}\text { Implanted Sapien } \\
\text { valve size }[\mathrm{mm}]\end{array}$ & $23(n=3), 26(n=11), 29(n=6)$ \\
\hline $\begin{array}{l}\text { Age at catheteriza- } \\
\text { tion (range) [years] }\end{array}$ & $27(8-58)$ \\
\hline Weight (range) [kg] & $69.565(28-101)$ \\
\hline BSA (range) $\left[\mathrm{m}^{2}\right]$ & $1.77(0.98-2.24)$ \\
\hline \multirow{2}{*}{$\begin{array}{l}\text { Indication for inter- } \\
\text { vention }\end{array}$} & Isolated RVOT stenosis $(n=4)$ \\
\hline & $\begin{array}{c}\text { Mixed stenosis and regurgitation } \\
(n=17, \text { mild } \mathrm{PI} n=5, \text { moderate } \mathrm{PI} n=4, \\
\text { severe } \mathrm{PI} n=8)\end{array}$ \\
\hline
\end{tabular}

$M$ - male, F-female, TOF-tetralogy of Fallot, RVOT-right ventricular outflow tract, BPV - bioprosthetic valve, TID - true internal diameter, PPVI-percutane ous pulmonary valve implantation, BSA - body surface arena, PI - pulmonary incompetence. tion and $16.5 \mathrm{mg} / \mathrm{dl}(11-27)$ afterwards (normal range: $7-25 \mathrm{mg} / \mathrm{dl})$. Mean serum creatinine of $0.77 \mathrm{mg} / \mathrm{dl}(0.41-$ 1.43) before the intervention and $0.78 \mathrm{mg} / \mathrm{dl}(0.49-1.45)$ after the intervention (normal range: $0.70-1.30 \mathrm{mg} / \mathrm{dl}$ ). Baseline renal biochemistry was not assessed in pediatric patients ( $<18$ years), but all patients had previously normal parameters of renal biochemistry documented.

\section{Perioperative imaging}

Sixteen patients underwent perioperative cross sectional imaging, 5 patients computed tomography (CT), and 12 patients cardiac magnetic resonance (CMR). One patient had both CT and MRI documented. Median time from cross sectional imaging assessment to catheter intervention was 76 days (3-505). Fusion overlay imaging (Vessel Navigator, Philips, Figure 2) was performed in 9 patients. Patients undergoing CMR had standard evaluation of RV function, neo-pulmonary valve regurgitation as well as measurements and qualitative descriptions of the RVOT, main pulmonary artery and branch pulmonary arteries, which were also assessed in those patients who had a CT. Coronary relationships were predominantly evaluated using preoperative imaging. Coronary proximity to the valve ring and the tips of the frame of the BPV, as well as quantification of the coronary distance from areas of significant calcification, were reported. Patients with $<5 \mathrm{~mm}$ distance between the nearest coronary artery and one of the aforementioned structures in either systole or diastole were reassessed intra-procedurally using aortic root, selective or 3D rotational coronary angiography during an RVOT

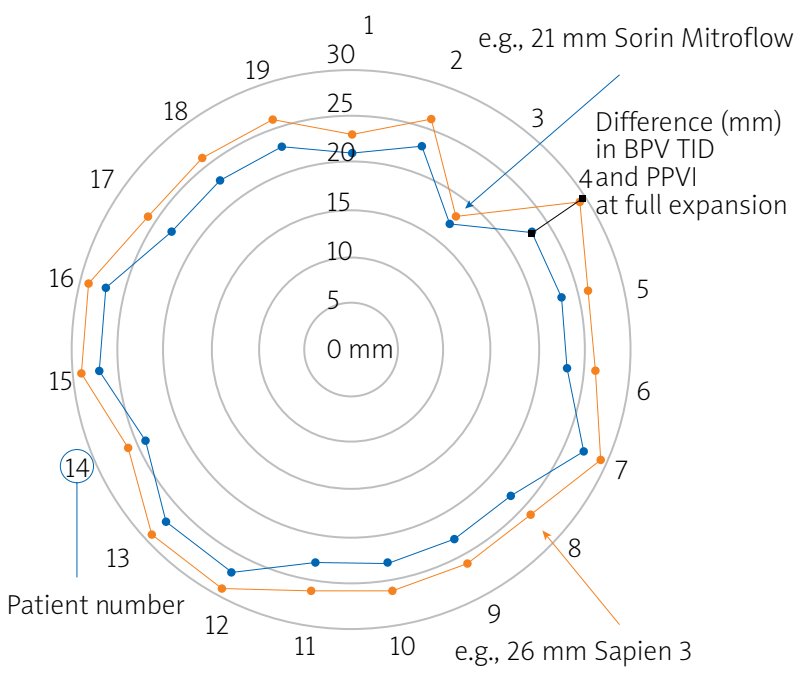

Relationship of TID of BPV to implanted valve size $(n=19)$ - True internal diameter $(\mathrm{mm})$ - Impanted valve size $(\mathrm{mm})$

Figure 2. Radar chart demonstrating the difference $(\mathrm{mm})$ between the true internal diameter (TID) of bioprosthetic valves (purple) (2) and the fully expanded implanted valve diameter (orange) in 19 patients (numbered) with BPV 
balloon interrogation. Patients without cross sectional imaging also had angiographic coronary evaluation intra-procedurally [3]. Intraprocedural coronary assessment was performed in 7 patients with a median volume of $8 \mathrm{ml}$ (range: 3-13) of contrast used. No patient had contrast used in assessment of the RVOT.

\section{Procedural details}

Our institutional protocol for assessment and implantation of PPVI is described in detail elsewhere [4, 5]. Twenty-one patients were identified for listing for minimal or zero-contrast PPVI. Vascular access for valve deployment was via a femoral venous approach in all patients. A standard right heart catheterization and hemodynamic assessment was performed in each case. Mean procedure time was $82.43 \pm 21.9 \mathrm{~min}$ (range: 48-126). Mean fluoroscopy time was $20.57 \pm 7.76$ min (range: $9.5-$ 40.7). Mean air kerma was $188.76 \pm 237.12 \mathrm{mGy}$ (range: 15-792). Mean total dose area product (DAP) was 23317 \pm 34556 mGy- $\mathrm{cm}^{2}$ (range: 454-120447). Contrast angiography of at least one coronary artery was performed in 7 patients due to an indeterminate preprocedural coronary relationship with the RVOT. Distal pulmonary arterial positioning was established using a balloon tipped catheter and exchange wire which allowed exchange for a stiff wire. Gore Dryseal sheaths, $65 \mathrm{~cm}$ in length, were positioned along the exchange wire and used to deliver the valve to the outflow tract. Sapien 3 (Edwards Lifesciences Corporation) transcatheter valves mounted on the commander delivery system (Edwards Lifesciences Corporation) were used in every case. The relationship of implantation valve size to BPV true internal diameter (TID) is demonstrated in Figure 3. Intracardiac echocardi- ography (ICE, Figure 4) was used to assess the newly implanted valve and the tricuspid valve along with confirming hemodynamic assessment. There was no planned intentional valve frame fracture in this cohort. An unintentional valve frame fracture was noted in one single patient on a pre-discharge X-ray film. Chest radiograph, transthoracic echocardiography and ECG were then performed $24 \mathrm{~h}$ after the procedure, prior to discharge.

\section{Hemodynamics}

Right ventricular pressure fell from a mean of $60 \pm 14$ $\mathrm{mm} \mathrm{Hg}$ (range: 35-93) before intervention to a mean of $36 \pm 10 \mathrm{~mm} \mathrm{Hg}$ (range: $20-55$ ) after (Figure 5) with a $p$-value of $<0.01$. Right ventricular to pulmonary artery (RVPA) gradient fell from a mean of $30 \pm 10 \mathrm{~mm} \mathrm{Hg}$ (range: 9-50) before the intervention to a mean of $4.35 \pm 4.6 \mathrm{~mm} \mathrm{Hg}$ (range: 0-15) after (Figure 6) with a $p<0.005$. Transthoracic echocardiography (TTE) estimated RVPA gradient fell from $43 \pm 16.9 \mathrm{~mm} \mathrm{Hg}$ (range: 14-68.9) pre-procedurally to $19.2 \pm 9.79$ (range: $1.9-36$ ) post-procedurally. Intracardiac echocardiographic (ICE) maximum velocity across the valve was $1.42 \pm 0.34 \mathrm{~m} / \mathrm{s}$ (range: $0.78-2$ ). No patients had more than trivial PI on the ICE assessment at the end of the procedure.

\section{Reintervention and complications}

One patient had a significant complication requiring re-intervention 3 months after discharge. He had a complex background with a diagnosis of Shone's complex requiring coarctation repair, Ross procedure, homograft implantation in the pulmonary position and transverse arch stenting. The patient had poor baseline kidney function exacerbated by biventricular cardiac dysfunction leading
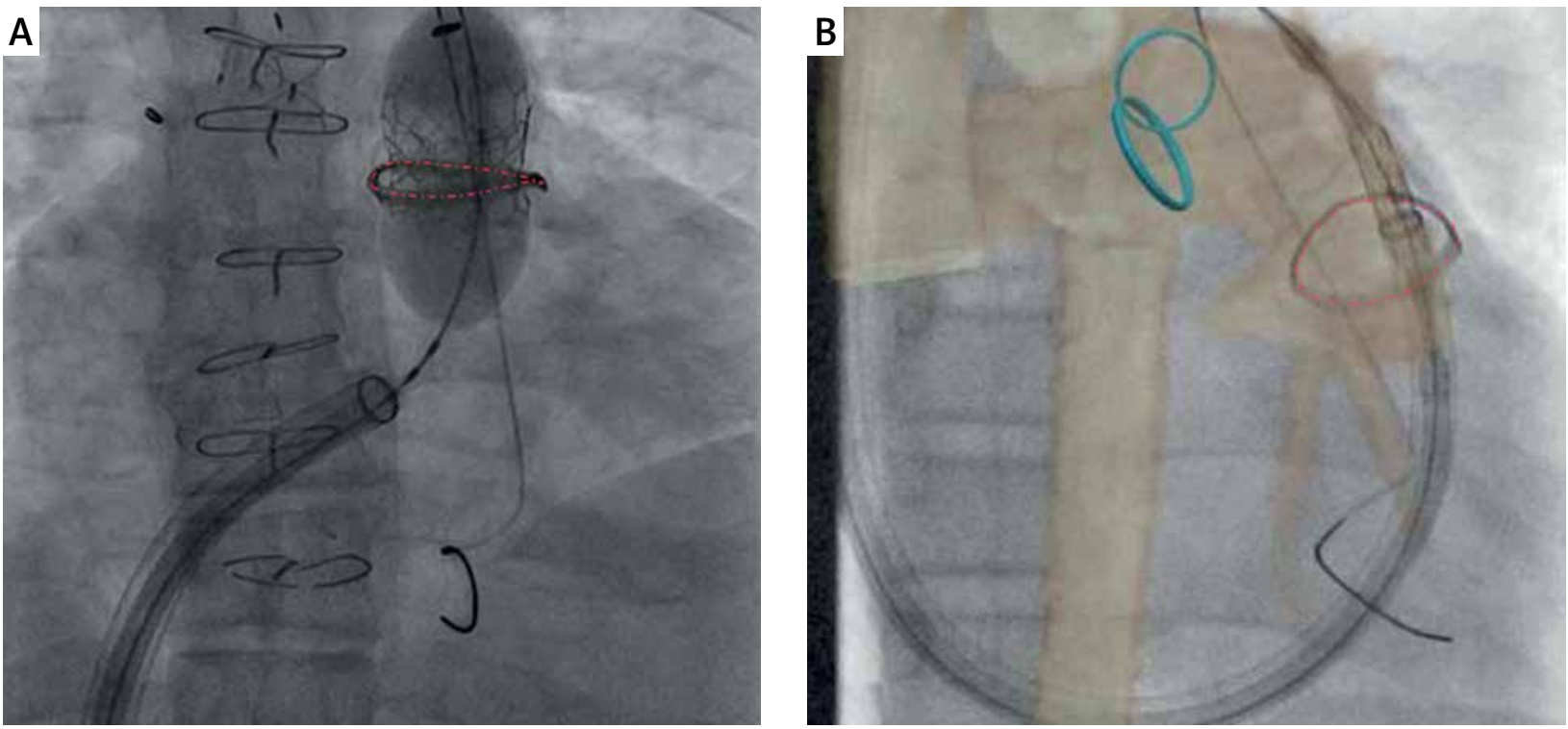

Figure 3. A - Sapien 3 Valve balloon expanded within Sorin Mitroflow BPV (red line). B - Philips Vessel Navigator overlaying CT data of the RVOT and branch PA's guiding positioning of an Edwards Valve within the Sorin Mitroflow (red line) 

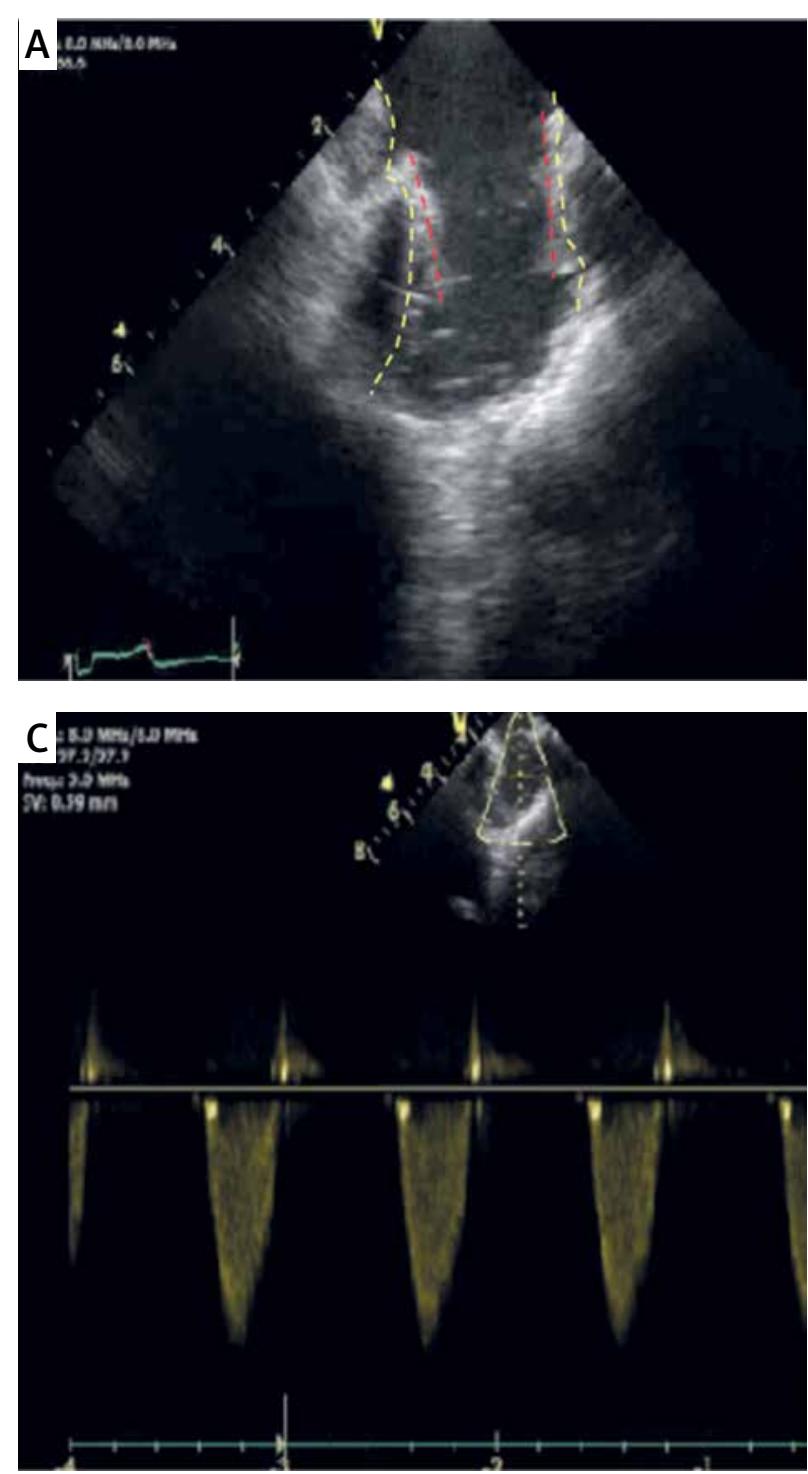

to cardiorenal syndrome. We had determined that contrast should be used judiciously and therefore, considering the careful pre-operative imaging evaluation using both CT and MRI, proceeded to implant his PPVI without pre- or post-procedural RVOT angiography. He re-presented 17 days after his valve implantation with dysphagia, dysphonia and shortness of breath, which were felt to be symptomatic of a slowly expanding pseudoaneurysm. At this point his renal failure and cardiovascular status had improved somewhat. CT angiography demonstrated an extensive hemomediastinum secondary to a small RVOT perforation with complete obliteration of esophageal patency and significant tracheal obstruction (Figure 7). We brought him to the catheterization laboratory and localized a small leak anteriorly at the proximal extent of the dilated RVOT. We placed a $39 \mathrm{~mm}$ long covered CP stent (B. Braun Inc.) which was expanded across this area using a $24 \mathrm{~mm}$ balloon and a $2^{\text {nd }} 23 \mathrm{~mm}$ Sapien valve was implanted within the stent. The pseudoaneurysm and its

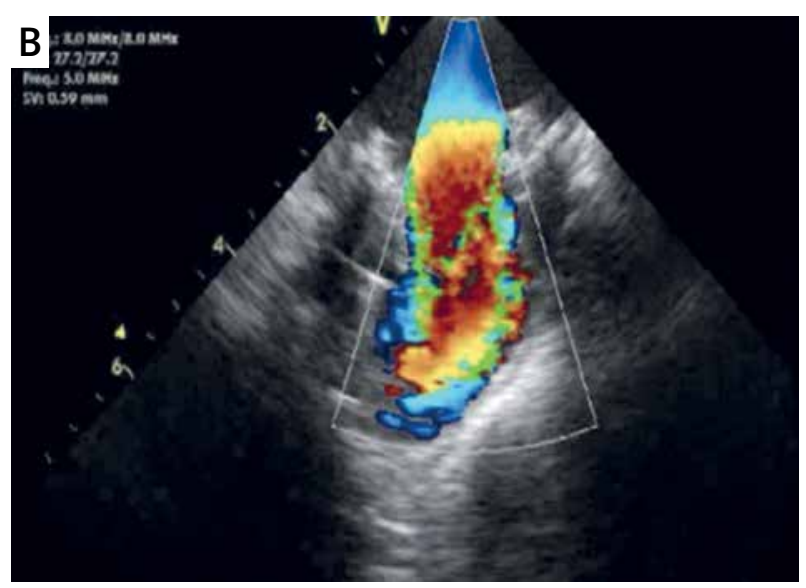

Figure 4. Intracardiac echocardiography to assess post-PPVI hemodynamics and function: $A$ - the $2 \mathrm{D}$ anatomical image of the pre-procedural RVOT (yellow line) and the newly implanted Edwards valve (red line), B - 2D color doppler assessment of the valve which infers high speed flow and turbulence through the center of the valve, C - a pulsed wave (PW) Doppler assessment through the new valve predicting the maximum velocity of flow (Vmax) through the valve

attendant symptoms eventually resolved. One patient remained an inpatient for 10 days following valve implantation to optimize medical management of her chronic respiratory disease prior to discharge. The prolonged admission time was not related to the PPVI. All other patients were discharged within $48 \mathrm{~h}$.

\section{Discussion}

Percutaneous pulmonary valve implantation was first performed by Bonhoeffer et al. [6] in London, United Kingdom in 2000 - the culmination of years of work developing the world's first dedicated transcatheter valve system, the Melody valve (Medtronic Inc. Minneapolis, $M N$ ). The bovine jugular prosthesis achieved US Food and Drug administration (FDA) approval in 2010. A variety of studies and series have been published over the last 15 years detailing the experience of operators and patients who have undergone transcatheter valve implantation in the RVOT [7-11], and generally this approach to 


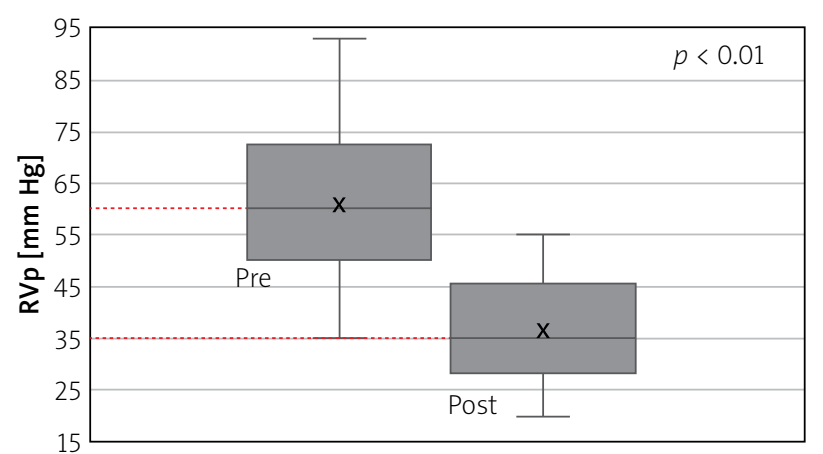

Figure 5. Right ventricular pressure (RVp) in $\mathrm{mm} \mathrm{Hg}$ before (pre) and after (post) percutaneous pulmonary valve implantation with statistical significance reported
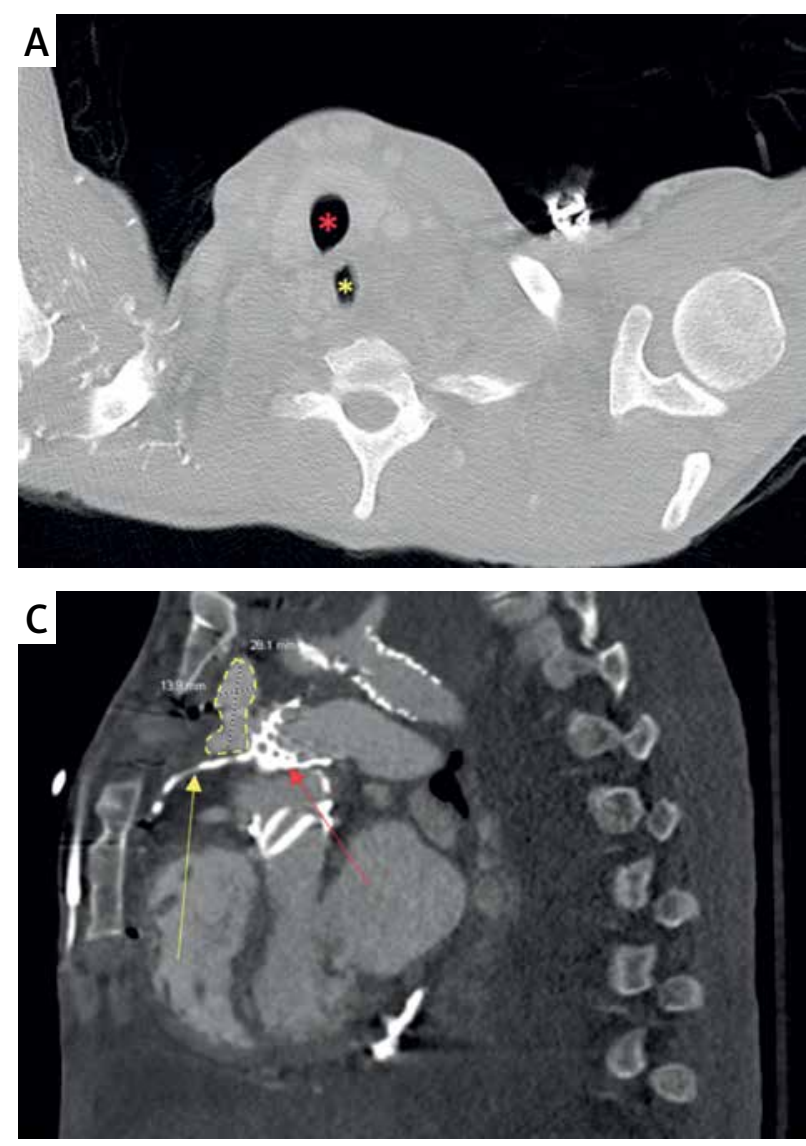

treatment is commended as being outstanding, with acceptable complication profiles and a limited requirement for surgical reintervention/bailout despite the off-label use of various devices [12].

Surgical conduits (e.g. Contegra, Medtronic), homografts (aortic, pulmonary) and bioprosthetic valve constructs (e.g. Sorin Mitroflow, CE Magna) are used to replace the pulmonary valve, following the almost inevitable failure of initial surgical interventions on the RVOT. Patient and material characteristics determine the long-

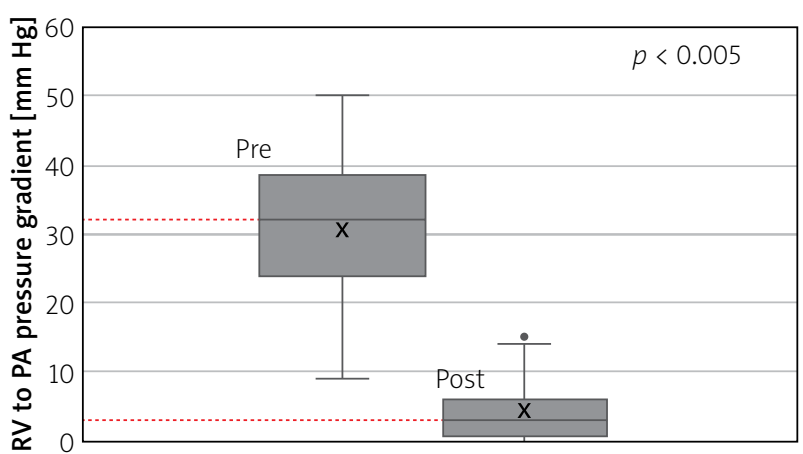

Figure 6. RV to PA pressure gradient in $\mathrm{mm} \mathrm{Hg}$ before and after percutaneous valve implantation (PPVI) with statistical significance reported

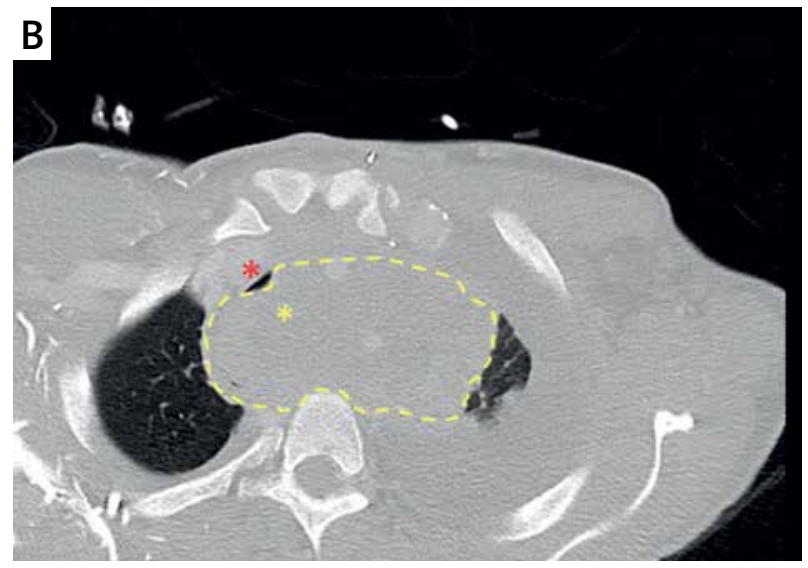

Figure 7. CT angiogram detailing: A - supracardinal patency of the airway and esophagus at the level of C2, caudal to this (B) there is a large organized hematoma (yellow line) at the level of aortic arch branching causing near obliteration of the bronchus (red asterisk) and complete esophageal obliteration (yellow asterisk). C - a sagittal section demonstrating a moderate sized pseudoaneurysm (dotted yellow line) at the point of interaction between the Sapien valve frame (red arrow) and the extensively calcified homograft (yellow arrow)

term behavior of this initial intervention and have a significant impact on the choice of devices that might be suitable for treating subsequent RVOT dysfunction.

Whilst the incidence of contrast-related reactions and renal dysfunction is low in patients under the age of 3 , there are considerations for older children and young adults with congenital heart disease with respect to its judicious use [13]. There are no predefined thresholds in children which guide the use of contrast agents with respect to kidney function [14]. Many pediatric patients will 
not have routine renal biochemistry and metabolic panels performed due to the anticipated normality of such results and perhaps due to the lack of guidance around their utility with our patient group in practice [15-17]. The older child, teenager and young adult population represent a majority of patients attending the cardiac catheterization laboratory and overwhelmingly represent the age group most commonly undergoing PPVI. Whilst many will have normal parameters of renal function on laboratory assessment, one must acknowledge the variety of insults on the developing kidneys of these young patients having come through congenital heart services over their early years [18]. Estimated glomerular filtration rate (eGFR) is calculated based on serum creatinine levels and used to categorize patients based on their renal function in large scale adult studies. Its use in pediatric practice is more limited due to its inability to detect early acute kidney injury (AKI) after renal insults such as intravascular contrast use $[17,19]$. A variety of effects contribute to the poor renal profile of young adults with treated and untreated congenital heart disease. One study on ACHD patients' kidney function detailed that half of all patients have significantly impaired renal function and $20 \%$ of those have moderate to severe impairment [15]. They are 18 times more likely to have renal impairment than age-matched controls in the general population. Moderate to severe impairment is seen more typically in patients with cyanosis and Eisenmenger's syndrome. Mortality is 3-fold higher in ACHD patients with moderately to severely impaired renal function. This presents an additional complexity to the ongoing care of these patients as survival continues to improve and an expanded population of ACHD patients with renal dysfunction requires more complex care [15]. Cautious use of contrast agents both inside and outside of the cardiac catheterization laboratory in both growing and grown up congenital heart patients is likely to be enforced more scrupulously in the future. Whilst the typical risk factors associated with contrast induced injury in patients with chronic kidney disease may not apply to the adult congenital heart disease group (diabetes mellitus, older age), other risk factors such as hypertension, congestive heart failure and use of nephrotoxic drugs do exist and should be considered when planning for contrast evaluation prior to and during cardiac catheterization [16].

A variety of methods are used to plan and work through the procedure of pulmonary valve implantation including a variety of imaging modalities which can be used to infer the characteristics and potential behaviors of the RVOT on intervention. Computed tomographic angiography (CTA) allows for both pre-procedural planning and intraoperative fusion imaging presenting the RVOT as a live 3D overlay during device placement and deployment $[5,20]$. Understanding the relationship of the coronary arterial course to the potential landing zone of the stent construct allows for suitability assessment and prevention of coronary compression during the procedure. We traditionally measure the shortest distance from the coronary artery to the BPV, the narrowest point in the RVOT or to the nearest projection of adjacent calcium. In the case of BPV, we measure the nearest distance from the coronary artery to the BPV frame. Patients with $<5 \mathrm{~mm}$ distance to the narrowing, calcification or BPV frame are usually considered for balloon interrogation of coronary compression. Measurements are taken in both systole and diastole in patients with electrocardiogram (ECG) gated imaging [21]. This may or may not be complemented by balloon interrogation of the RVOT with coronary assessment during the procedure [22]. $\mathrm{MRI}$ can also be used preoperatively to assess the coronary to RVOT relationship and, similarly to $\mathrm{CT}$, can be used as overlay fusion imaging for intraprocedural image guidance. Both can provide imaging data on extra coronary fat, which can provide a predictive marker for relative safety of valve deployment [23]. Coronary arterial distance, regional tissue characteristics, local RVOT substrate, and the anticipated degree of enlargement of the outflow tract may determine the optimal landing zone for a device, and all of these features may be interpretable from pre-procedural cross sectional imaging. All patients in our study who had absent, indeterminate or non-recent ( $>1$ year) imaging data for coronary relationship assessment had a limited contrast assessment of the coronary tree with balloon inflation. Aortic root distortion and relationships of the aortic root to the implantation zone may be described and predicted from cross sectional imaging, but in our experience, neither alter the course of treatment in patients with BPV, who usually experience minimal displacement of the RVOT during valve implantation [4]. Stiff sheaths and wires used in the implantation of PPVI can distort the anatomy as it relates to the pre-calibrated fusion imaging. It is our experience that anatomical distortion is less common in patients with non-native outflow tracts, particularly those with heavy calcification or indeed with surgically implanted bioprosthetic valve apparatus. These tissues and inventory can provide a stable anchor point for wires and sheaths as they curve through the outflow tract. Where anatomical distortion is exaggerated and image overlay significantly interrupted we recommend repeat registration of the image fusion software after wire and/or sheath placement, prior to valve implantation.

Routine practice is such that further imaging is obtained at the time of catheterization with fluoroscopy, balloon interrogation, biplane angiography and 3 dimensional rotational angiography (3DRA). All of these modalities can be performed to further evaluate the RVOT substrate and optimal landing zone for percutaneous pulmonary valve implantation. However, in certain cases, such additional assessments may not contribute ultimately to decision making and may or may not provide any information to support the technical aspects of the 
case. All of the above adjuncts and modalities increase operative time, radiation exposure and indeed contrast exposure. They may additionally increase cost and complications. Published experience on the use of fusion imaging implies that it can diminish operative time, radiation exposure and contrast exposure in certain substrates of disease and specific procedures [23]. In summary, contrast angiographic assessment of the RVOT in patients with BPV is still used despite much of the information for safely carrying out the procedure being readily available both pre- and intra-procedurally. Uniquely, patients with bioprosthetic valves have pre-defined surgical apparatus within the RVOT with a predetermined size, internal lumen and mechanical characteristics that are reasonably well understood. The behavior of bioprosthetic valves in percutaneous 'valve-in-valve' implantation is usually more predictable than PPVI in a homograft or RVPA conduit [24]. They provide a somewhat predictable landing zone for a percutaneous valve and their frames can often be safely fractured [25] to improve the available space for expansion of valve-in-valve implants. It is our contention that many of these valves provide adequate landmarks for device implantation with or without the adjunct of fusion imaging. There therefore may be less of a role for RVOT angiography in specific patient groups, whilst small doses of contrast can be used to evaluate indeterminate coronary profiling on preoperative imaging. All practitioners should reserve the right to re-evaluate the RVOT using angiography to assess for an unexpected complication after valve deployment. This may however become less relevant in patients with 'predictable' outflow tracts such as those with BPV.

The ideal candidate for this pathway is a patient with a dysfunctional bioprosthetic surgical valve of an adequate diameter that will not require intentional fracture to accommodate an appropriately sized percutaneous valve. The Edwards Sapien 3 transcatheter bovine pericardial valve has in our experience been the valve most suited to implantation in BPV interventions, mainly as it does not require pre-stenting and has a length which matches and adequately covers the frames of most dysfunctional surgical prostheses without redundancy. Patients, where possible, will have cross sectional imaging in the form of CT or MRI which describes the relationship between the coronary arteries and the potential valve landing zone. These imaging modalities may be fused with live fluoroscopic images to provide additional information for valve deployment with the RVOT. Where image fusion is not available and pre-operative cross sectional imaging is indeterminate with respect to coronary relationships to the RVOT, a small dose of contrast can be used intraoperatively with or without balloon interrogation to assess the coronary relationship to the landing zone of the valve. On valve deployment, hemodynamic assessment and intracardiac echocardiography provide adequate data to inform the operator of successful valve deployment and function. These parameters are then correlated with postoperative transthoracic echocardiographic assessment of the RVOT. The reintervention and complication rate of $4.7 \%$ in our series relates to 1 patient, who, in retrospect, lies outside the suggested scope for contrast-free valve implantation. Renal protection strategies such as pre-hydration, sodium bicarbonate administration or $\mathrm{N}$-acetylcysteine administration could have been employed to justify a contrast angiographic evaluation of this patient's RVOT [16, 26]. The most extensive report to date on patients with Sapien 3 PPVI reports complication rates of $10 \%$ accounting for all RVOT substrates [27]. Our ongoing practice remains such that in the immediate post-PPVI evaluation of patients with RVOT conduits and calcified homografts, we perform RVOT angiography to assess for contained or uncontained tissue rupture. This case demonstrates the importance of contrast angiography in the assessment of RVOT interventions in patients with complex RVOT anatomy, particularly when expanding conduits or homografts. In such cases, our ability to recognize certain complications without high quality angiography is very limited.

In select patient groups, contrast-free RVOT evaluation should be a welcome venture considering the advent of fusion imaging as a more routine approach to congenital cardiac catheterization, particularly for patients requiring percutaneous pulmonary valve implantation [22].

This was a retrospective analysis and thus the intrinsic biases of these are present. The timing of intervention was variable and based on broad hemodynamic indications. Pre-operative cross sectional imaging was not performed in all patients. Fusion imaging was not used in all cases even when cross sectional imaging was available, somewhat in part due to the use of several different catheterization laboratories, one of which did not have image fusion capabilities. Advanced cross sectional imaging evaluation and fusion imaging may not be present in all centers performing these interventions. Renal functional parameters were not available for all patients and assumptions were made based on previously documented results remote from the time of the study. There is a small sample size with limited follow-up data for the purposes of resolving long term success of the intervention.

\section{Conclusions}

Contrast-free assessment of the RVOT at the time of PPVI is technically possible in patients with straightforward BPVs that do not require intentional frame fracture or enlargement of their outer diameter by other means. The availability of fusion imaging using pre-operative image data sets provides the capacity to expand the number and anatomical complexity of patients to whom we can offer this approach. Improved understanding of 
how preoperative imaging assessment can fully predict coronary interactions with percutaneous valves and provide a complete overview of the RVOT for the purposes of valve implantation will expand the number of patients and complexity of RVOT substrate that can benefit from low- or no-contrast PPVI. Given our experience, this pathway should be limited to those patients whose substrate is that of a previously implanted bioprosthetic valve of known diameter. Patients with RVOT conduits and calcified homografts are likely to need angiographic RVOT evaluation at the time of PPVI to evaluate for injury.

As outlined above, there is a dearth of literature on renal disease in CHD patients, particularly important in the ageing ACHD population. As the complexity of our patient group continues to intensify and survival improves, we will encounter more patients who require vigilance and caution with respect to contrast exposure. The cumulative effects over time of ischemia-reperfusion injury, systemic cyanosis, impaired renal perfusion and nephrotoxic drugs, as well as normal ageing, dictate that contrast agents must be used scrupulously to prevent the evolution of chronic kidney disease in patients with CHD.

\section{Conflict of interest}

Dr Morgan is a proctor for Edwards Lifesciences Corporation. The remaining declare no conflict of interest.

\section{References}

1. van der Ven JPG, van den Bosch E, Bogers AJCC, Helbing WA. Current outcomes and treatment of tetralogy of Fallot. F1000Res 2019; 8: F1000.

2. Ozkok S, Ozkok A. Contrast-induced acute kidney injury: a review of practical points. World J Nephrol 2017; 6: 86-99.

3. Valvein ValveAorticapp[Internet].[cited20200ct1].Availablefrom: https://www.pcronline.com/PCR-Publications/PCR-mobileapps/Valve-in-Valve-Aortic-app

4. Malone L, Fonseca B, Fagan T, et al. Preprocedural risk assessment prior to PPVI with CMR and cardiac CT. Pediatr Cardiol 2017; 38: 746-53.

5. Goreczny S, Zablah J, McLennan D, et al. Multi-modality imaging for percutaneous pulmonary valve implantation - getting serious about radiation and contrast reduction. Adv Interv Cardiol 2019; 15: 110-5.

6. Bonhoeffer P, Boudjemline Y, Saliba Z, et al. Percutaneous replacement of pulmonary valve in a right-ventricle to pulmonary-artery prosthetic conduit with valve dysfunction. Lancet 2000; 356: 1403-5.

7. Kenny D, Rhodes JF, Fleming GA, et al. 3-year outcomes of the Edwards SAPIEN transcatheter heart valve for conduit failure in the pulmonary position from the COMPASSION multicenter clinical trial. JACC Cardiovasc Interv 2018; 11: 1920-9.

8. Morgan GJ, Sadeghi S, Salem MM, et al. SAPIEN valve for percutaneous transcatheter pulmonary valve replacement without "pre-stenting": a multi-institutional experience. Catheter Cardiovasc Interv 2019; 93: 324-9.

9. Cabalka AK, Hellenbrand WE, Eicken A, et al. Relationships among conduit type, pre-stenting, and outcomes in patients un- dergoing transcatheter pulmonary valve replacement in the prospective north american and european melody valve trials. JACC Cardiovasc Interv 2017; 10: 1746-59.

10. Cheatham JP, Hellenbrand WE, Zahn EM, et al. Clinical and hemodynamic outcomes up to 7 years after transcatheter pulmonary valve replacement in the US melody valve investigational device exemption trial. Circulation 2015; 131: 1960-70.

11. Husain J, Praichasilchai P, Gilbert Y, et al. Early european experience with the venus $p$-valve ${ }^{\circledR}$ : filling the gap in percutaneous pulmonary valve implantation. Eurolntervention 2016; 12: e643-51.

12. Chatterjee A, Bajaj NS, McMahon WS, et al. Transcatheter pulmonary valve implantation: a comprehensive systematic review and meta-analyses of observational studies. J Am Heart Assoc 2017; 6: e006432.

13. Callahan MJ, Servaes S, Lee EY, et al. Practice patterns for the use of iodinated IV contrast media for pediatric CT studies: a survey of the society for pediatric radiology. Am J Roentgenol 2014; 202: 872-9.

14. Dillman JR, Strouse PJ, Ellis JH, et al. Incidence and severity of acute allergic-like reactions to IV nonionic iodinated contrast material in children. Am J Roentgenol 2007; 188: 1643-7.

15. Tkaczyk M, Tomczyk D, Jander A, et al. Glomerular filtration decrease after diagnostic cardiac catheterisation in children with congenital cardiac malformation - the role of serum creatinine, cystatin C, neutrophil gelatinase and urine output monitoring. Adv Interv Cardiol 2018; 14: 67-74.

16. Hung YM, Lin SL, Hung SY, et al. Preventing radiocontrast-induced nephropathy in chronic kidney disease patients undergoing coronary angiography. World J Cardiol 2012; 4: 157-72.

17. Benzer M, Alpay H, Baykan Ö, et al. Serum NGAL, cystatin C and urinary NAG measurements for early diagnosis of contrast-induced nephropathy in children. Ren Fail 2016; 38: 27-34.

18. Konstantinos D, Gerhard-Paul D, Evdokia K, et al. Prevalence, predictors, and prognostic value of renal dysfunction in adults with congenital heart disease. Circulation 2008; 117: 2320-8.

19. Schwartz GJ, Work DF. Measurement and estimation of GFR in children and adolescents. Clin J Am Soc Nephrol 2009; 4: 1832-43.

20. Chung R, Taylor AM. Imaging for preintervention planning transcatheter pulmonary valve therapy. Circ Cardiovasc Imaging 2014; 7: 182-9.

21. Tezza M, Witsenburg M, Nieman K, et al. Cardiac CT to assess the risk of coronary compression in patients evaluated for percutaneous pulmonary valve implantation. Eur J Radiol 2019; 110: 88-96.

22. Morray BH, McElhinney DB, Cheatham JP, et al. Risk of coronary artery compression among patients referred for transcatheter pulmonary valve implantation a multicenter experience. Circ Cardiovasc Interv 2013; 6: 535-42.

23. Goreczny S, Moszura T, Dryzek P, et al. Three-dimensional image fusion guidance of percutaneous pulmonary valve implantation to reduce radiation exposure and contrast dose: a comparison with traditional two-dimensional and three-dimensional rotational angiographic guidance. Neth Heart J 2017; 25: 91-9.

24. Asoh K, Walsh M, Hickey E, et al. Percutaneous pulmonary valve implantation within bioprosthetic valves. Eur Heart J 2010; 31: 1404-9.

25. Shahanavaz S, Asnes JD, Grohmann J, et al. Intentional fracture of bioprosthetic valve frames in patients undergoing valve-invalve transcatheter pulmonary valve replacement. Circ Cardiovasc Interv 2018; 11: e006453. 
26. Ronco F, Tarantini G, McCullough PA. Contrast induced acute kidney injury in interventional cardiology: an update and key guidance for clinicians. Rev Cardiovasc Med 2020; 21: 9-23.

27. Shahanavaz S, Zahn EM, Levi DS, et al. Transcatheter pulmonary valve replacement with the Sapien prosthesis. J Am Coll Cardiol 2020; 76: 2847-58. 